\title{
Silicon photonic resonator sensors and devices
}

\author{
Lukas Chrostowski $^{a}$, Samantha Grist $^{a}$, Jonas Flueckiger ${ }^{a}$, Wei Shi $^{a}$, Xu Wang $^{a}$, Eric Ouellet $^{b}$, Han \\ Yun $^{a}$, Mitch Webb ${ }^{a}$, Ben Nie ${ }^{a}$, Zhen Liang ${ }^{a}$, Karen C. Cheung ${ }^{a}$, Shon A. Schmidt ${ }^{c}$, Daniel M. \\ Ratner $^{c}$, and Nicolas A. F. Jaeger ${ }^{a}$ \\ ${ }^{a}$ Department of Electrical and Computer Engineering, and \\ ${ }^{b}$ Department of Chemical and Biological Engineering, \\ University of British Columbia, Vancouver, British Columbia, Canada \\ ${ }^{c}$ Department of Bioengineering, University of Washington, Seattle, Washington, USA
}

\begin{abstract}
Silicon photonic resonators, implemented using silicon-on-insulator substrates, are promising for numerous applications. The most commonly studied resonators are ring/racetrack resonators. We have fabricated these and other resonators including disk resonators, waveguide-grating resonators, ring resonator reflectors, contra-directional grating-coupler ring resonators, and racetrack-based multiplexer/demultiplexers.

While numerous resonators have been demonstrated for sensing purposes, it remains unclear as to which structures provide the highest sensitivity and best limit of detection; for example, disc resonators and slot-waveguide-based ring resonators have been conjectured to provide an improved limit of detection. Here, we compare various resonators in terms of sensor metrics for label-free bio-sensing in a micro-fluidic environment. We have integrated resonator arrays with PDMS micro-fluidics for real-time detection of biomolecules in experiments such as antigen-antibody binding reaction experiments using Human Factor IX proteins. Numerous resonators are fabricated on the same wafer and experimentally compared. We identify that, while evanescent-field sensors all operate on the principle that the analyte's refractive index shifts the resonant frequency, there are important differences between implementations that lie in the relationship between the optical field overlap with the analyte and the relative contributions of the various loss mechanisms.

The chips were fabricated in the context of the CMC-UBC Silicon Nanophotonics Fabrication course and workshop. This yearlong, design-based, graduate training program is offered to students from across Canada and, over the last four years, has attracted participants from nearly every Canadian university involved in photonics research. The course takes students through a full design cycle of a photonic circuit, including theory, modelling, design, and experimentation.
\end{abstract}

Keywords: silicon photonics, lab-on-a-chip, microfluidics, evanescent optical field sensors, optical waveguides, disk resonators, ring resonators, slot waveguides

\section{INTRODUCTION}

Silicon nanophotonic devices have great potential as highly integrated, low-cost biosensors for medical, point-of-care (POC) and home healthcare diagnostic applications. Recent advances in the silicon photonic biosensor literature have demonstrated clinically relevant sensitivities, and an expanded repertoire of biocompatible chemistries capable of interrogating a variety of biological molecules. Additionally, by leveraging existing CMOS fabrication processes, silicon photonic sensors offer significant advantages in economies of scale over traditional biosensing platforms, allowing for the integration of thousands of sensors on a single millimetre-scale chip. These sensors are poised to displace traditional assays that rely upon multi-step liquid handling processes, significant user intervention, and cumbersome bench-top instrumentation. However, numerous challenges must be overcome to realize a fully integrated silicon photonic biosensing platform, including understanding the optimal sensor characteristics, fluidic and analyte control and on-chip lasers and detectors. This paper discusses important sensor performance metrics in aqueous environments similar to saliva, serum or blood, essential for medical diagnostic applications.

Evanescent field sensors such as Surface Plasmon Resonance (SPR) or planar waveguide based sensors are amongst the most popular optical detection techniques for sensitive and label free biomolecular detection. ${ }^{1}$ Compared to other sensing methods, optical sensors methods have the advantages of high sensitivity, no physical contact between sensor and detector, immunity to electromagnetic interference, and multiplexed interrogation. Waveguides with dimensions smaller

Laser Resonators, Microresonators, and Beam Control XIV, edited by Alexis V. Kudryashov, Alan H. Paxton, Vladimir S. Ilchenko, Lutz Aschke, Kunihiko Washio, Proc. of SPIE Vol. 8236, 823620 - (C) 2012 SPIE · CCC code: 0277-786X/12/\$18 - doi: 10.1117/12.916860 
than the free-space wavelength of light have a strong evanescent field at the waveguide surface extending a few hundred nanometers into the surrounding media. Any interaction with molecules in proximity or surface bound will change the effective refractive index of the guided mode, which leads to a shift in the optical spectrum of the device. These sensors have shown promise for real time detection of biomolecules because of their sensitivity towards surface binding events.

In the case of a straight waveguide-based sensor, such as a Mach-Zehnder, the interaction is limited by the physical length of the sensing waveguide. The degree of interaction of the evanescent field and the sample for a ring resonator is limited by the number of roundtrips by the light inside the ring and the footprint of the sensing area can be reduced without losing sensitivity. In general, high quality factor (Q) resonators are advantageous for reduced spectral noise of the sensor, ${ }^{2}$ leading to an improved detection limit.

Optical sensors based on ring and racetrack resonators have been proposed and developed using a variety of materials and for a variety of applications such as chemical sensing ${ }^{3,4}$ and sensing of biological molecules such as antibodies or antigens. ${ }^{5-10}$ In particular, the optical, label-free detection of biomolecules is a topic of considerable interest. ${ }^{11,12}$ The sensitivity of a ring resonator is dependent on the overlap of the evanescent field and the sample. ${ }^{2}$ In a strip waveguide most of the field is contained inside the core and only a small portion propagates outside in the media. Most experiments have focused on TE-like polarized waveguide modes, likely since low loss TE waveguides have been available. ${ }^{13}$ However, the TM-like waveguide mode has been shown to offer $3 \mathrm{X}$ improved sensitivity (S, nm/RIU) ${ }^{14}$ as compared to the TE mode since it has a larger evanescent field component above the waveguide.

To enhance the sensitivity, several alternative resonator structures have been proposed and demonstrated. Slot waveguide resonators ${ }^{4,15-19}$ have shown even further enhanced sensitivity since there is much more optical field contained in the fluid. Other structures proposed and/or demonstrated include disk resonators, ${ }^{20,21}$ disk resonators containing a fluorescent material, ${ }^{22} 1 \mathrm{D}$ photonic crystals with holes in waveguide, ${ }^{23} 2 \mathrm{D}$ photonic crystals, and Bragg grating cavities. ${ }^{24}$ Ring sensors have also been integrated on the end of an optical fibre. ${ }^{25}$

There has been significant interest in integrating optical sensors with microfluidic channels specifically for the detection of bio-species as well as other molecules. ${ }^{26}$ Several groups have integrated ring resonator sensors with microfluidic channels ${ }^{7,8}$ including multiplexing the slot-ring resonator sensors such that only one optical input is used for multiple sensors. ${ }^{8,27}$ Resonator sensors have been arranged in folded-waveguide configurations to make the best possible use of space on the optical device. ${ }^{28}$ To advance biomedical applications, inkjet printing of chemicals ${ }^{29}$ on the resonant sensors has been demonstrated.

\subsection{Biomedical Applications of Silicon Photonics}

Silicon photonic microring resonators, like those described previously, show great promise as label-free sensing and analysis tools in biomedical research, drug development, homeland security, food safety, environmental monitoring and healthcare in POC and low-resource settings. ${ }^{30}$ Notably, the sensitivity and multiplexing capability of silicon photonic biosensors has advanced to the point where the technology is now competitive with the enzyme-linked immunosorbent assay (ELISA), the current gold standard for clinical assays. Conventional ELISAs utilizes a multi-well microtiter plate containing immobilized antibodies that bind to a specific analytes of interest. ELISA-based analysis depends upon multiple time-consuming rinse cycles, the consumption of a significant volume of reagent, and the addition of an enzyme-labeled antibody for optical detection via formation of a chromophore or chemiluminescent reaction. While sensitive and reliable at clinically relevant levels, the ELISA involves costly reagents and a skilled operator, rendering it impractical for distributed, low-resource, and home healthcare settings. Label-free biosensors have been investigated as a competitive alternative to the ELISA. ${ }^{1}$ Similar to the ELISA, label-free biosensors leverage a functionalized surface to selectively capture targets species. However, unlike ELISA, label-free sensors directly detect the presence of analyte without additional reagents or labels, eliminating the need for multiple rinse steps and a secondary probes, and dramatically decreasing assay time.

In medicine, accurately diagnosing a disease state often requires the detection and quantification of multiple biological markers (proteins, nucleic acids, lipids, carbohydrates and small organic biomolecules). Silicon photonic sensors facilitate simultaneous multi-analyte detection by interrogating matrixed sensor arrays on a single chip, referred to as multiplexed detection. ${ }^{31}$ For example, a single-marker assay for prostate-specific antigen (PSA) to detect prostate cancer possessed a meager $65 \%$ sensitivity and $35 \%$ specificity. ${ }^{32}$ By contrast, a multi-marker protein panel improved the sensitivity and specificity to $83 \%$ and $97 \%$, respectively. This result highlights the inherent advantage of multiplexed detection in medical diagnostics. 
While a fully integrated, label-free silicon photonics biosensor has yet to be realized commercially, the technology has established its utility for medical diagnostics at clinically relevant sensitivities. ${ }^{1}$ Washburn et al. have demonstrated simultaneous analysis of multiple biomarkers on a single microring sensor array, successfully quantifying PSA, $\alpha$-fetoprotein (AFP), carcinoembryonic antigen (CEA), tumor necrosis factor- $\alpha$ (TNF- $\alpha$ ), and interleukin-8 (IL-8) at clinical concentrations. ${ }^{33}$ Like proteins, MicroRNAs (mRNAs) play a role in a variety of disease states, including cancer, heart disease, and central nervous system disorders, and can be used as markers for early disease detection and diagnosis. Using siliconbased photonic sensors, Qavi et al. demonstrated a multiplexed mRNA diagnostic capability by discriminating between single nucleotide polymorphisms of the let-7 family in concentrations of $150 \mathrm{fmol}$ with minimal sample preparation. ${ }^{34} \mathrm{In}$ addition, Ramachandran et al. have reported that whole bacteria can be successfully detected using microring resonators. ${ }^{6}$ These examples illustrate the transformative potential of label-free silicon-photonic sensors.

At present, the aforementioned biosensing examples rely on external lasers and detectors, which limit their application for distributed multiplexed sensing and diagnosis. On-chip detectors capable of interrogating thousands of functionalized sensors are necessary to realize a fully distributable diagnostic device. Towards this goal, Yan et al. recently demonstrated an optical sensor fabricated in a commercially available $0.35 \mu \mathrm{m}$ CMOS technology with integrated electronic detectors. ${ }^{35}$ Kirk et al. developed a method to rapidly functionalize individual rings in a 50 x $50 \mu \mathrm{m}$ footprint using a commercially available non-contact piezo printer that can be scaled to print multiple chips in parallel. ${ }^{29}$ Finally, Hochberg et al. reported on the benefits of a shared-wafer fabrication consortium which offers low-cost prototyping through commercially available CMOS chip fabrication facilities. ${ }^{36}$ These strategies, coupled with low-cost LED light sources point to the significant role that a fully integrated label-free, silicon-based nanophotonic diagnostic device could play in both POC diagnostics and distributed healthcare.

\subsection{Sensor Performance - Criteria}

This section describes the most important performance criteria which are useful for comparing different sensor geometries. The metrics are based on the work of White ${ }^{2}$ and Yoshie $^{37}$ particularly for micro-resonator sensors. The most important are:

1. Sensitivity (S), which is the slope of wavelength shift versus bulk refractive index unit change [nm/RIU], also sometimes known as Responsivity (R). In a resonator, the wavelength shift is described by: ${ }^{38}$

$$
\frac{\Delta \lambda}{\lambda}=\frac{\Delta n_{\text {fluid }}}{n_{g}} \frac{\partial n_{\text {eff }}}{\partial n_{\text {fluid }}} \text {. }
$$

The contributions to the shift are from: a) the shift in the analyte's index of refraction ( $\left.\Delta n_{\text {fluid }}\right)$, b) the material and waveguide dispersion $\left(n_{g}\right)$ - a change in wavelength causes a further change in effective index, and c) the change in index slightly changes the mode profile resulting in the mode's effective index change (susceptibility, $\partial n_{\text {eff }} / \partial n_{\text {fluid }}$ ). The same effect is found in the temperature dependence of waveguide-based resonators. ${ }^{39}$ In high-contrast silicon photonic devices, $n_{g}$ is typically $4-5$, and $\partial n_{\text {eff }} / \partial n_{\text {fluid }}$ is approximately 0.15 (for strip and ridge waveguides). In contrast free-space propagation would have $\partial n_{\text {eff }} / \partial n_{\text {fluid }}=1$.

The sensitivity is defined as

$$
S=\frac{\Delta \lambda}{\Delta n_{\text {fluid }}},
$$

and the normalized sensitivity (useful for comparing different wavelength resonators) is

$$
S^{\prime}=\frac{S}{\lambda}=\frac{\Delta \lambda}{\lambda} \frac{1}{\Delta n_{\text {fluid }}} .
$$

2. Q factor, which is the number of oscillations before the energy has decayed to the 1/e value. A high Q-factor means that light is present in the resonator longer and interacts more with the analyte. It is also advantageous in reducing the spectral noise of the sensor, ${ }^{2}$ which improves the limit of detection. The resonator Q can be found from the total distributed loss of the resonator (in $m^{-1}$, in $d B / m$, or in terms of the material complex index of refraction, $n+i k$ ),

$$
Q=\omega_{0} \frac{\mathcal{E}}{d \mathcal{E} / d t}=\omega_{0} \tau_{p}=\frac{\omega_{0}}{\alpha_{\left[m^{-1}\right]} \cdot \frac{c}{n_{g}}}=\frac{2 \pi \cdot n_{g}}{\lambda_{0} \cdot \alpha_{\left[m^{-1}\right]}}=\frac{2 \pi \cdot n_{g} \cdot 4.34}{\lambda_{0} \cdot \alpha_{[d B / m]}}=\frac{n_{g}}{2 \cdot k} .
$$


These losses originate from waveguide scattering, material absorption (waveguide and analyte), waveguide radiation loss, mode mismatch losses, etc. Equation 4 is useful to identify the intrinsic Q for a resonator with a particular waveguide propagation loss, e.g., $3 \mathrm{~dB} / \mathrm{cm}$ waveguides yield intrinsic $\mathrm{Q}$ values of 258,000 at $1550 \mathrm{~nm}$; if this resonator was critically coupled, the $\mathrm{Q}$ value is reduced by a factor of two and becomes 129,000 .

3. Limit of Detection (LOD, or Detection Limit, DL) which is the minimum detectable refractive index unit change [RIU]. The detection limit can be defined two ways: 1) change in index corresponding to one resonator linewidth $\left(\Delta n_{\text {min }}\right)$ - this is useful to compare the intrinsic device performance, and 2) a system detection limit $\left(\Delta n_{m i n, s y s}\right)$ which corresponds to the index change for a standard deviation in the measurement noise (here, the wavelength reading noise). The highest performing systems have a detection limit on the order of $10^{-7}$ RIU. ${ }^{40}$

The limit of detection is calculated by ${ }^{37}$

$$
\Delta n_{\min }=L O D=\frac{1}{Q S^{\prime}}
$$

4. The limit of detection can also be expressed in a biological perspective, e.g., in terms of the adsorbent's thickness.

\subsection{Comparison of Experimental Sensitivity and Detection Limit}

Here, we compare several silicon photonics evanescent field sensors operating at $1550 \mathrm{~nm}$. We compare the intrinsic detection limit, i.e., the change in index corresponding to one resonator linewidth $\left(\Delta n_{\text {min }}\right)$. This comparison allows one to compare the intrinsic device performance, independent of the read-out method, electronics and noise. The comparison includes previous literature on ring/racetrack resonators, ${ }^{5,10,25,41}$ slot resonators, ${ }^{7}$ and $1 \mathrm{D}$ photonic crystals. ${ }^{23}$ It also includes the experimental results presented in Section 2. For each device, the normalized Sensitivity (S') and Q are identified (in water) and the results are plotted in Figure 1.

The data points with the highest limit of detection are found towards the upper-right of the plot, and the limit of detection is identified by the contour lines using Equation 5. The highest reported limit of detection is for the TM-like mode strip waveguide, ${ }^{41}$ with the slot waveguides and photonic crystals having the worst performance.

Since this plot is for volume index of refraction changes, not included is the single-molecule detection work of Vahala ${ }^{21}$ where the high-Q cavity results in locally heating the molecule and inducing a thermo-optic effect which results in enhanced wavelength shifts. SPR sensors ${ }^{42}$ are also not included. They work on the same principle as resonators, namely an evanescent field interacts with the sample, but the evanescent field originates from a surface plasmon in a metal film. Comparing dielectric and SPR sensors: a) SPR typically has a slightly longer field decay (200 nm vs. $100 \mathrm{~nm})$, b) sensitivities are much higher, but the limit of detection is similar. As an example of a commercial SPR system, the Biacore $\mathrm{X}$ has a noise of $0.6 \mathrm{RU}$ (where $1 \mathrm{RU}$ is defined as $10^{-6} \mathrm{RIU}$ ), ${ }^{43}$ hence a detection limit of $0.6 \times 10^{-6} \mathrm{RIU}$.

\section{EXPERIMENTAL RESULTS ON THE SAME WAFER}

Silicon photonic devices were fabricated using $193 \mathrm{~nm}$ lithography by IMEC accessed via CMC Microsystems and ePIXfab. All designs described in this section were fabricated on the same wafer to aid in the direct comparison of the resonators. SEM images of the fabricated devices are shown in Figs. 3, 5, and 6.

\subsection{Lab-on-a-chip, PDMS Microfluidics}

The concept of a lab-on-a-chip combines sample handling and analysis in one single chip, thereby offering the possibility of a portable analysis platform. Picoliter sample volumes can be handled inside micrometer scale fluidic channels using valves and pumps integrated into silicon or polymer chips. ${ }^{44}$ It is of great interest to include sensor technology into these lab-on-chip devices to automate parallel processing and therefore lower cost and increase throughput. Ksendzov et al. clamped a flow cell down onto the sensor chip. ${ }^{45}$ They achieved sealing by using an O-ring and pressure. However they are not able to expose individual resonators with different analytes. Washburn et al. and Luchansky et al. used a laser cut Mylar gasket aligned over top of the microring arrays to define microfluidic channels. ${ }^{1,9}$ They closed the channels off with a Teflon lid and sandwiched the different layers between aluminum chip holders. Because mass transport is diffusion limited, they are not suitable to determine dynamics of fast reactions, e.g. binding events of proteins. Moreover, in order to be able to run several experiments with different analytes or different concentrations in parallel individual resonators need 


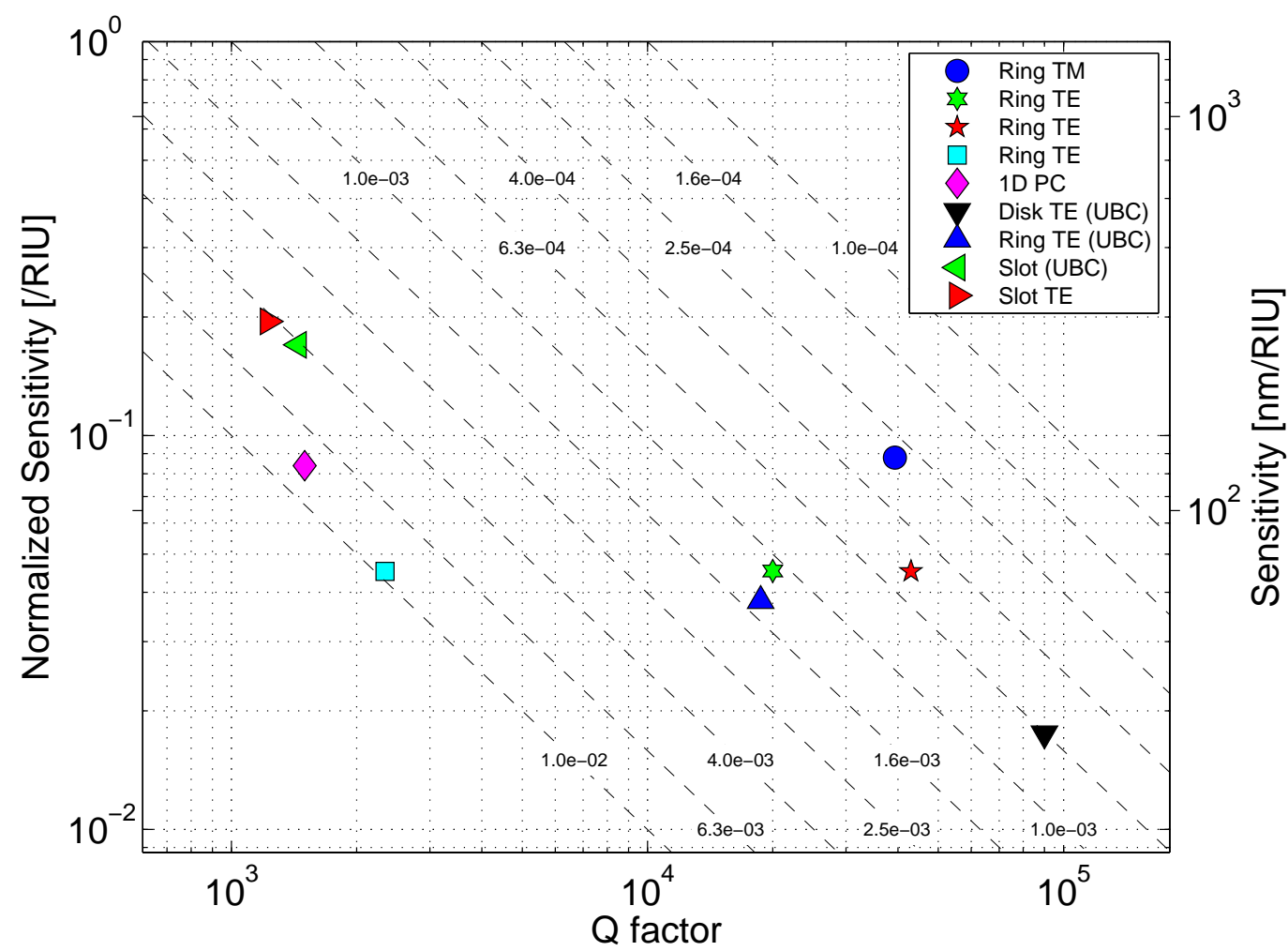

Figure 1. Summary of experimental results for Limits of Detection (LOD) for linear $1550 \mathrm{~nm}$ silicon photonic biosensors. Here, LOD is calculated for a noise equivalent to one resonator linewidth, which is useful to compare the intrinsic device performance. The diagonal lines correspond to LOD values of $\Delta n_{\min }=10^{-2}$ to $10^{-4}$. The right y-axis is the sensitivity, Eq. 2, while the left y-axis is the sensitivity normalized to the wavelength, Eq. 3.

to be addressed and exposed. Carlborg et al. used a microfluidic channel network in poly(dimethylsiloxane) (PDMS), with a separate fluid channel to each sensor for sample delivery. ${ }^{8}$

PDMS is the most widely used polymer in the field of microfluidics. It is a biocompatible, transparent, rubber-like polymer and can be easily patterned using soft lithography, a well established fabrication method. ${ }^{46}$ The mold masters were fabricated with standard photolithography techniques on SU-8 2075 (MicroChem, USA). Through replica molding the patterns were transferred onto PDMS. The uncured PDMS (Sylgard 184, Dow Corning USA) was poured onto the mold master to a thickness of about $1 \mathrm{~cm}$, degassed to remove air bubbles, and cured at $80^{\circ} \mathrm{C}$ for $2 \mathrm{~h}$ on a hotplate. The hydrophobic surface properties can be altered in an oxygen plasma by replacing some of the surface methyl groups (CH3) by a hydroxyl groups $(-\mathrm{OH})$. This activated surface can form covalent siloxane bonds ( $\mathrm{Si}-\mathrm{O}-\mathrm{Si}$ ) when in contact with glass or silicon substrates, forming an irreversible seal. However, we have found that we obtain sufficient sealing between the silicon chips and PDMS by performing reversible bonding. After punching access holes for the fluidic inlet and outlets the PDMS layer and the resonator substrate were aligned using a stereo microscope and bonded to each other. The PDMS channels were $100 \mu \mathrm{m}$ wide, $100 \mu \mathrm{m}$ tall, with a pitch of $300 \mu \mathrm{m}$. The bonded chips and measurement setup is shown in Fig. 2.

\subsection{Optical Setup}

The optical test setup for the fabricated nanophotonics chips consisted of a tunable laser source (Agilent 81682A, Agilent Technologies, Inc., USA) with an output wavelength range from $1460 \mathrm{~nm}$ to $1580 \mathrm{~nm}$; a polarization maintaining optical fibre was aligned with the fibre grating coupler to inject light into the SOI waveguides; the output light was collected with a single-mode optical fiber and measured with an optical power sensor (Agilent 81635A, Agilent Technologies, Inc., USA); the temperature of the substrate was kept constant with a Peltier element and a temperature controller (LDC501, Stanford Research System, USA) in a closed feedback loop configuration (typically 1-10 mK stability). A microscope system was 


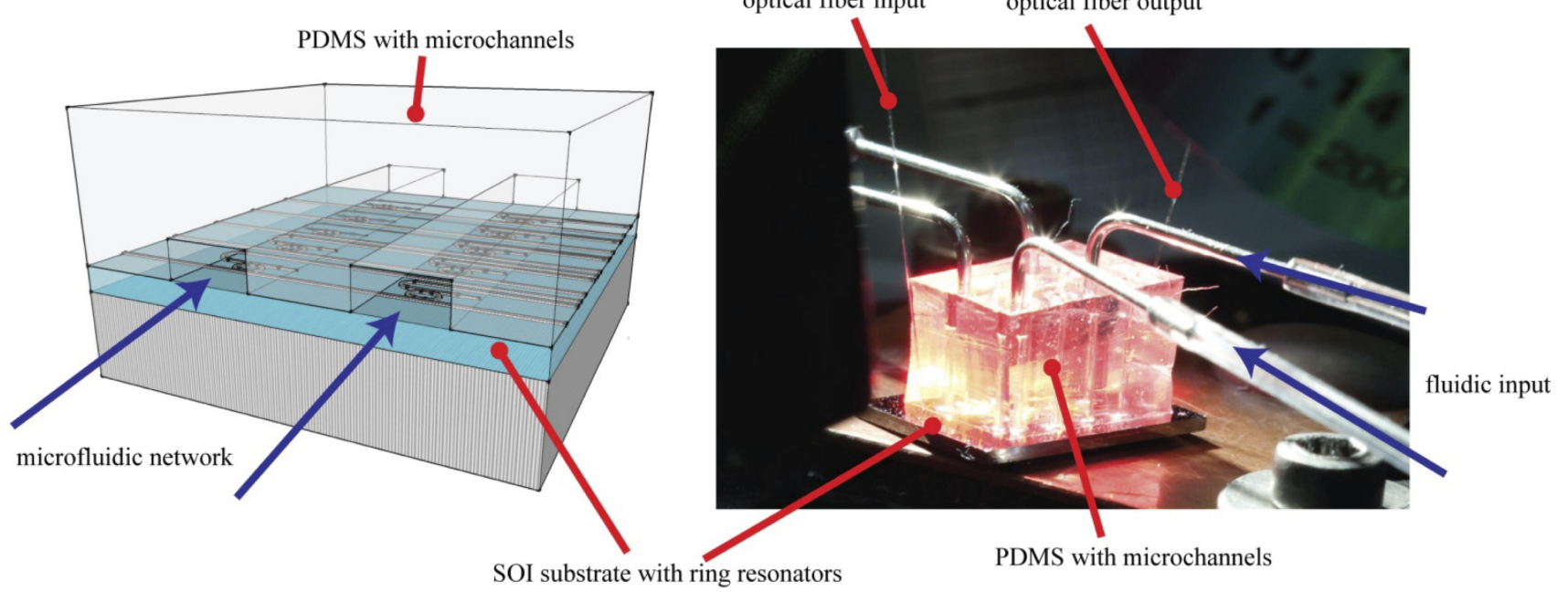

Figure 2. The measurement setup: ${ }^{48}$ the optical input fibre is connected to a tuneable laser source with wavelength at around $1550 \mathrm{~nm}$ and the output optical fibre is connected to an optical power sensor. The measurements are performed on a temperature controlled stage. Fluidic tubing are connected to a syringe pump.

used for fibre alignment. A MATLAB script was used to sweep the laser wavelength in order to acquire the transmission spectra for the various devices (sweep interval 20s with 10,000 data points).

For calibrating the sensors (sensitivity, Q), fluids of varying refractive index were flowed through the microfluidic channels under positive pressure, exposing the resonators to different immersion media refractive indices. Varying concentrations of glycerin in water were used as these varying known refractive indices. ${ }^{47}$ The positions of the resonance peaks were dynamically tracked by continuously sweeping the laser and curve fitting the spectrum. The wavelength tracking also proves useful when employing the resonators for biosensing applications, as it provides useful visual feedback when attaching proteins or antibodies to the resonators and is helpful to identify problems in the system (for example, a bubble in the channel).

\subsection{Ring resonators}

Racetrack resonators are designed following Rouger et. $\mathrm{al}^{49}$ and using the Lumerical MODE Solutions eigen-mode solver. ${ }^{50}$ The resonators were designed for critically-coupled double-bus configurations with parameters calculated using the MODE Solutions simulated material losses, the MODE Solutions simulated bend losses (radiation and mode-mismatch from straight to bent waveguide), and the straight-waveguide scattering losses from previous findings as well as the literature ( $4 \mathrm{~dB} / \mathrm{cm}$ for the strip waveguides) and $12 \mathrm{~dB} / \mathrm{cm}$ for the slot waveguides. ${ }^{7}$ The round-trip resonator losses were used to calculate the coupling lengths, where the coupling coefficients were calculated from the effective indices of the coupler super-modes.

Devices with both strip and slot waveguides were fabricated. Two of the fabricated chips were analyzed by SEM imaging and FIB cross-sectioning, as shown in figure 3. FIB cross-sectioning showed that the waveguides were narrower at their tops, with sidewall angles of approximately $9.5^{\circ}$.

\subsection{Slot waveguide ring resonator}

Mode profiles and modelling results for the slot waveguide design are shown in Fig. 4. Figure 4d presents the simulated immersion medium index sensitivity versus simulated waveguide base width. This is simulated to take into account the IMEC exposure dose sweep that was applied across the wafer, and which results in changes in the waveguide thickness. The changes also result in changes in the slot waveguide gap, which leads to changes in the sensitivity. The sensitivity decreases with increasing waveguide width, as a result of the increasing optical confinement of the mode within the silicon and a decreasing slot width. The measured sensitivity of $345 \mathrm{~nm} / \mathrm{RIU}$ is comparable to the $298 \mathrm{~nm} / \mathrm{RIU}$ reported for a similar design (with a similarly low Q factor of 330) in SOI by Claes et al. ${ }^{7}$ 

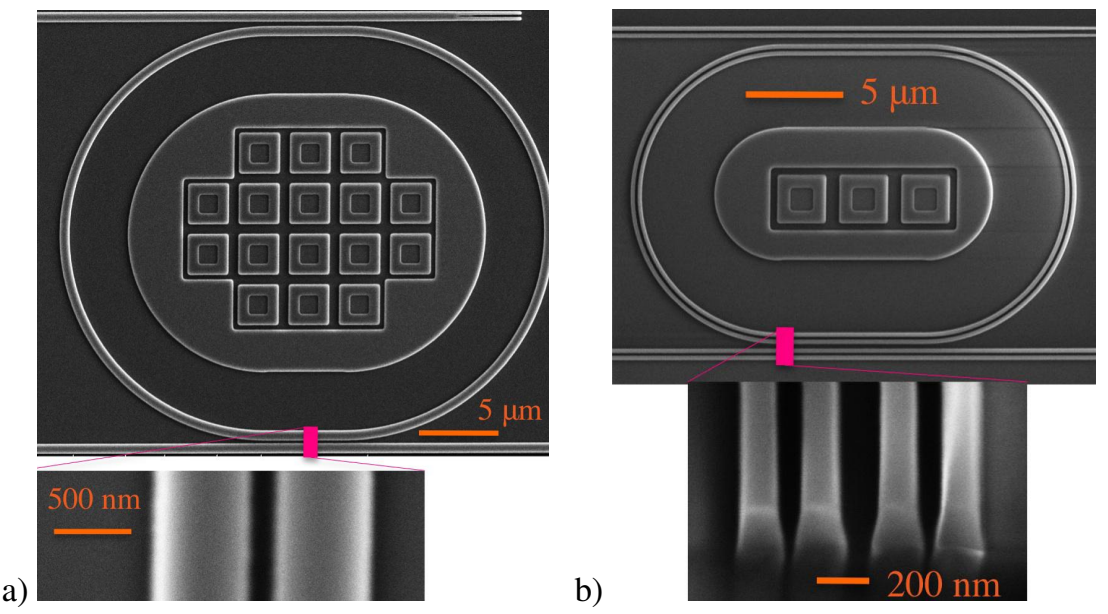

Figure 3. a) SEM images of strip waveguide ring resonators. The inset shows an SEM image of the highlighted coupler region. b) SEM images of slot waveguide ring resonators. The inset shows an SEM image of an FIB cross-section of the through port coupler; the slots in these waveguides are well-defined, all the way through the $220 \mathrm{~nm}$ of silicon.

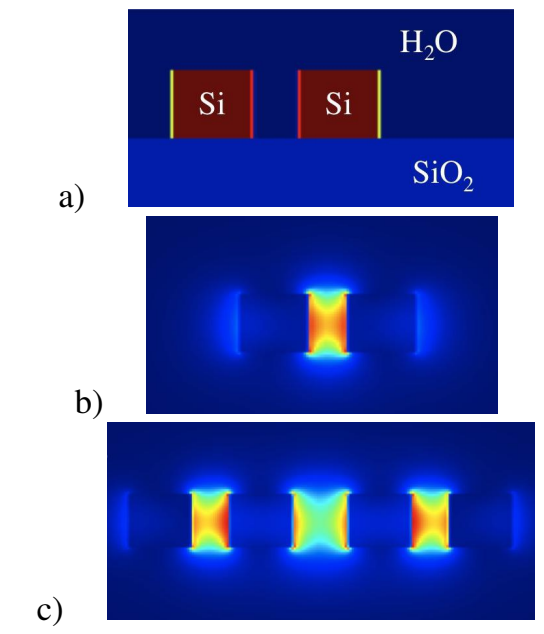

d)

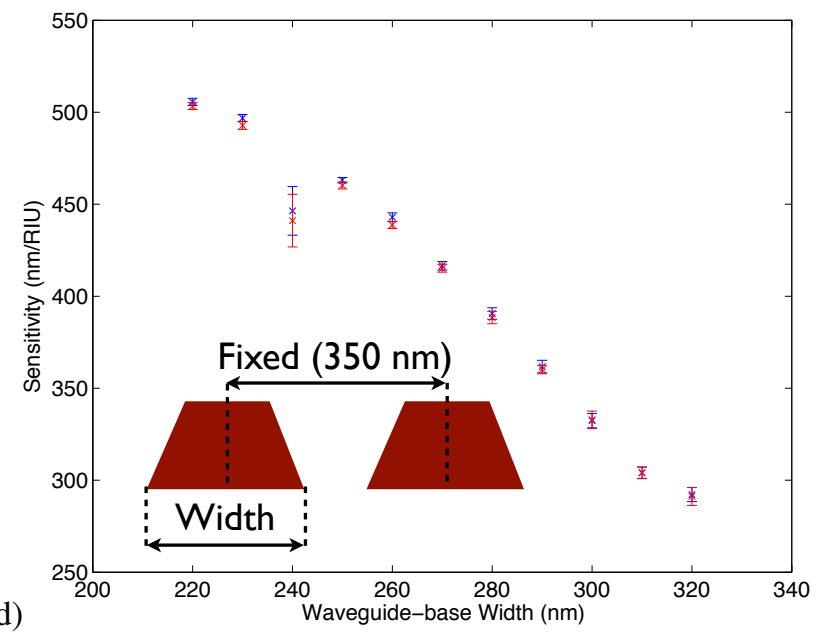

Figure 4. Simulations for the slot waveguide geometry: b) Material index profile. c) Electric field distribution for straight waveguide. d) Coupling super-mode 1 between parallel identical slot waveguides. d) Simulation results of immersion medium refractive index sensitivity vs. waveguide width for slot waveguide racetrack resonators. The blue points plot the sensitivity to a change from $0 \%$ to $2 \%$ glycerine, while the red points plot the sensitivity to a change from $2 \%$ to $4 \%$ glycerine.

\subsection{Bragg Grating resonators}

Bragg gratings, as well as quarter-shifted grating cavities were fabricated on the wafer. ${ }^{51}$ It is expected that the sensitivity of the grating will be equivalent to its straight waveguide counterpart. ${ }^{24}$ The quarter-shifted Bragg grating resonators demonstrated high-Q values of 100,000 with air cladding. The transmission spectrum of the device and SEM image are shown in Fig. 5.

\subsection{Disk resonators}

Ultra-compact, high-Q disk resonators have been theoretically investigated and experimental demonstrated. ${ }^{52-54}$ We use a similar geometry and size of disk ${ }^{54}$ in this investigation.

We discuss two approaches to modelling disk resonators which we have verified to have excellent agreement. The first method is to do a full 3D FDTD simulation of the entire disk. ${ }^{55}$ This is possible since the geometry of the disk is relatively small. The objectives of the simulation are: a) find the resonant modes of the disk, for a given polarization, and b) find the sensitivity (nm/RIU). The simulation includes the disk, the oxide, and the water, surrounded by PML boundary conditions. 
a)

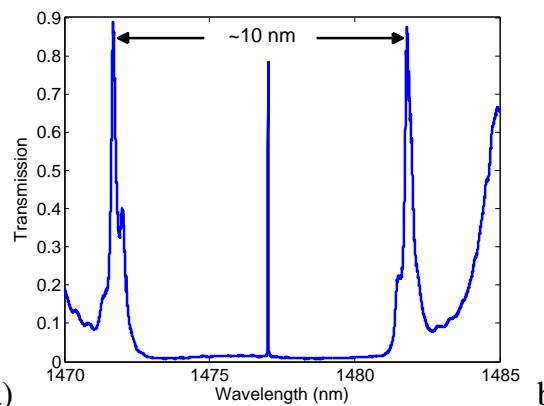

b)
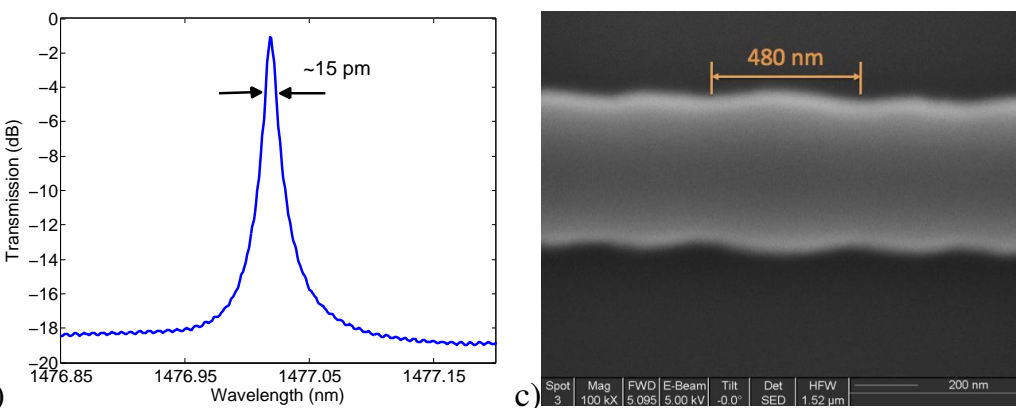

Figure 5. a) Measured transmission spectrum of the quarter-wave phase-shifted gratings, (b) enlarged plot around the transmission resonance, c) SEM image of the fabricated device, showing the quarter-shifted section.

The disk is excited by several dipole sources placed inside the disk. In order to find the sensitivity, we perform several FDTD simulations with a variation of cladding index of refraction. We then inspect the spectrum of the cavity and observe the shift in the cavity modes (nm), versus the applied changes in refractive index (RIU).
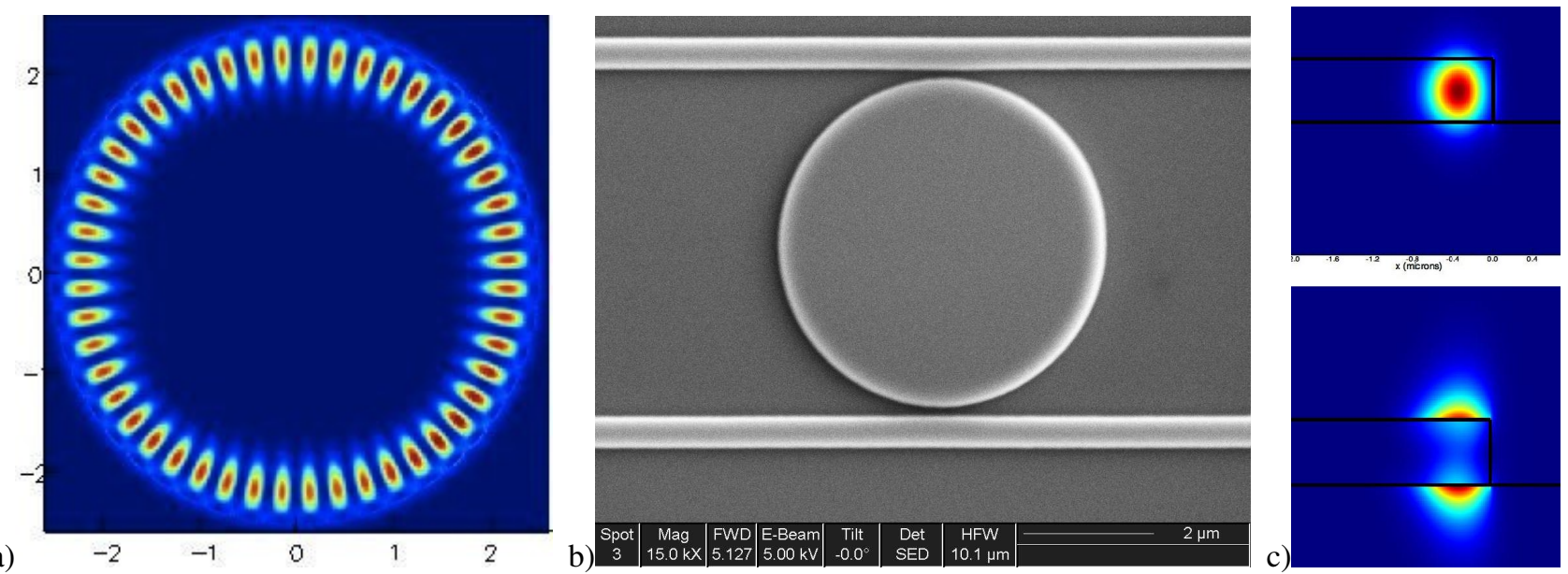

Figure 6. a) 3D FDTD calculation showing the top-view TE mode profile of a $2.5 \mu \mathrm{m}$ disk. Counting the number of nodes (48) indicates that this is mode \#24. b) SEM image of the disk. c) Mode calculation for the cross-section of the disk, TE mode (top), TM mode (bottom). All simulation plots are for the E-field intensity.

The second approach is similar to a conventional waveguide mode calculation, ${ }^{50}$ where we calculate the disk's crosssection mode profile, effective index, group index and loss for the lowest order TE and TM modes as a function of wavelength. The radius of the disk is included in the simulation by defining the bend radius as the actual radius of the disk. The advantage of this approach is that a much finer mesh can be employed since it is a 2D simulation, hence the accuracy can be improved. Similar to the 3D FDTD simulations, we vary the cladding index and record the shift in effective index. Next, we calculate the resonant modes by using simple Fabry-Perot theory, namely we find solutions where the round-trip phase is an integer multiple of $2 \pi$, using $2 \pi M=2 \pi n_{\text {eff }}(\lambda) L / \lambda$, where $L=2 \pi r$ is the circumference of the disk. We re-write this as

$$
M \frac{\lambda}{2 \pi r}=n_{\mathrm{eff}}(\lambda)
$$

and plot the results of the left-hand-side and right-hand-side of the equation in Figure 7a. The intersection of the $n_{\text {eff }}(\lambda)$ line with the mode number lines provides the solution(s) to the equation and yields the resonant wavelength(s). This technique can further be used to find the modes of different sized disks by recalculating the $n_{\text {eff }}$ versus wavelength graphs and re-plotting the above.

The loss of the each mode is also calculated by the mode solver, including the water absorption. For TM modes, the 

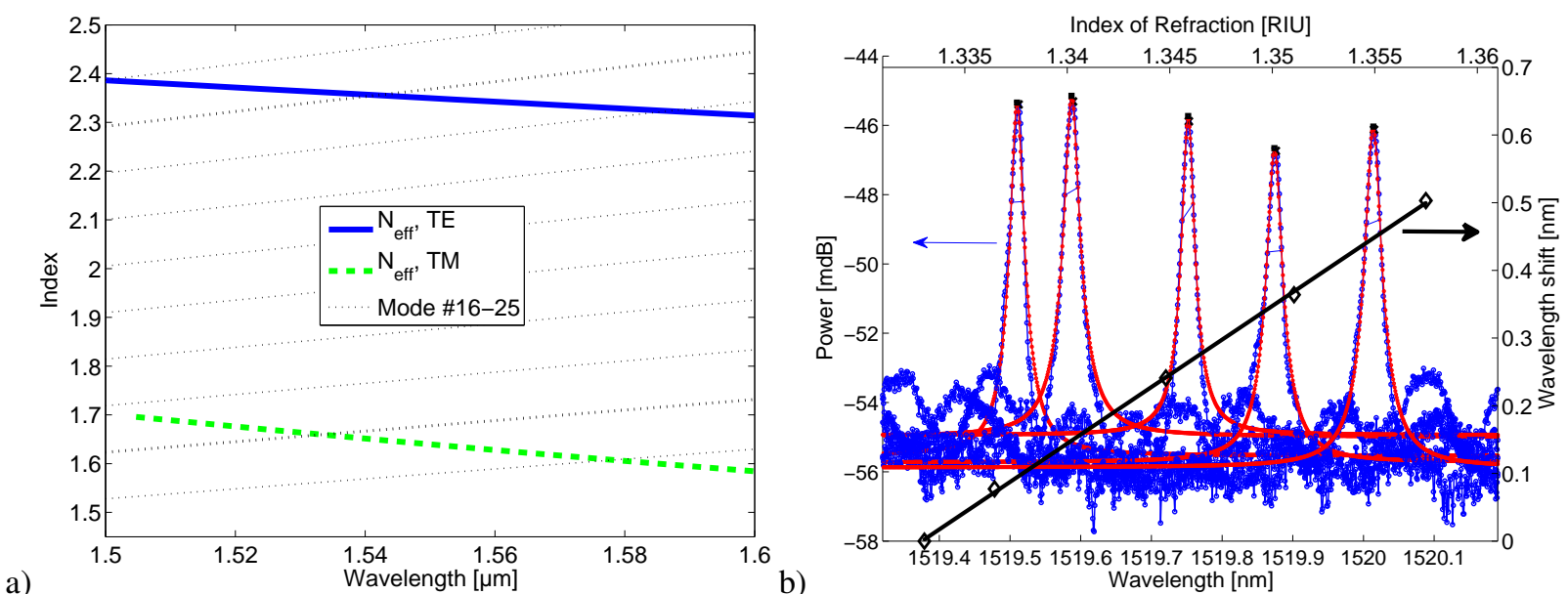

Figure 7. a) Effective index of the $2.5 \mu \mathrm{m}$ disk resonator modes versus wavelength. Upward trending lines are the required effective index values to achieve a resonant condition for mode numbers $m=16$ up to $m=25$. b) Experimental performance of a $5 \mu m$ disk resonator in water. The optical spectra (bottom and left axes) for the resonator show a Q of between 80,000 and 100,000. Varying the water index of refraction (top axis) by changing the concentration of glycerine changes the resonant wavelength (right axis); the sensitivity is $27.1 \mathrm{~nm} / \mathrm{RIU}$.

radiation loss cannot be neglected. ${ }^{54}$ In particular, the TM mode $2.5 \mu \mathrm{m}$ disk is impractical since the radiative losses are $600 \mathrm{~dB} / \mathrm{cm}$. Hence, larger disks are required for TM operation.

Experimental results for a disk resonator are shown in Figure 7b. The Q value in water is approximately 90,000, while the sensitivity is $27.1 \mathrm{~nm} / \mathrm{RIU}$.

\section{DISCUSSION AND ANALYSIS}

\subsection{Fundamental Limit for Water-Based Sensors}

We are interested in the fundamental limit of detection based on the water absorption and index change. We consider an ideal Fabry-Perot cavity and assume that the light travels entirely in the water, with no other loss mechanism other than water absorption. In this case $\partial n_{\text {eff }} / \partial n_{\text {fluid }}=1$. Using Eq. 1, this yields

$$
\frac{\Delta \lambda}{\lambda_{0}}=\frac{\Delta n_{\text {fluid }}}{n_{g}}
$$

or a sensitivity at $\lambda=1550 \mathrm{~nm}$ of

$$
S_{\text {ideal }}=\frac{\Delta \lambda}{\Delta n_{\text {fluid }}}=\frac{\lambda_{0}}{n_{g}}=1550 / 1.33=1165 \mathrm{~nm} / R I U
$$

Note that this fundamental limit applies to dielectric evanescent sensors. It also applies to grating-based SPR sensors, ${ }^{56}$ but not prism-coupled ones (which can achieve much higher $\mathrm{nm} / \mathrm{RIU}$ values).

Applying Eq. 4 for water absorption at $1550 \mathrm{~nm}\left(9.6 \mathrm{~cm}^{-1}\right)$ we find the intrinsic $\mathrm{Q}$ is limited to 5,500. The intrinsic $\mathrm{Q}$ for water is plotted versus wavelength in Fig. 8a. The Q is calculated from the water absorption spectrum ${ }^{57}$ using Eq. 4. This assumes that the light travels entirely in the water, as in an ideal Fabry-Perot cavity. Hence, $\mathrm{S}=1165 \mathrm{~nm} / \mathrm{RIU}$, and the limit of detection is $2.4 \times 10^{-4} \mathrm{RIU}$. This fundamental limit is included in Fig. 9. The intrinsic limit of detection is plotted versus wavelength in Fig. 8b, using Eqs. 4, 5 and 8. Also included is a curve with additional waveguide scattering loss of 5 $\mathrm{dB} / \mathrm{cm}$ at $1550 \mathrm{~nm}$ (based on previous measurements ${ }^{58}$ ), scaled using Rayleigh's $1 / \lambda^{4}$ dependance for other wavelengths. Finally, assuming a typical ${ }^{37}$ system readout sensitivity improvement of $100 \mathrm{X}$, the system's limit of detection would be $2.4 \times 10^{-6} R I U$ at $1550 \mathrm{~nm}$, and is plotted in Fig. $8 \mathrm{~b}$ versus wavelength. 

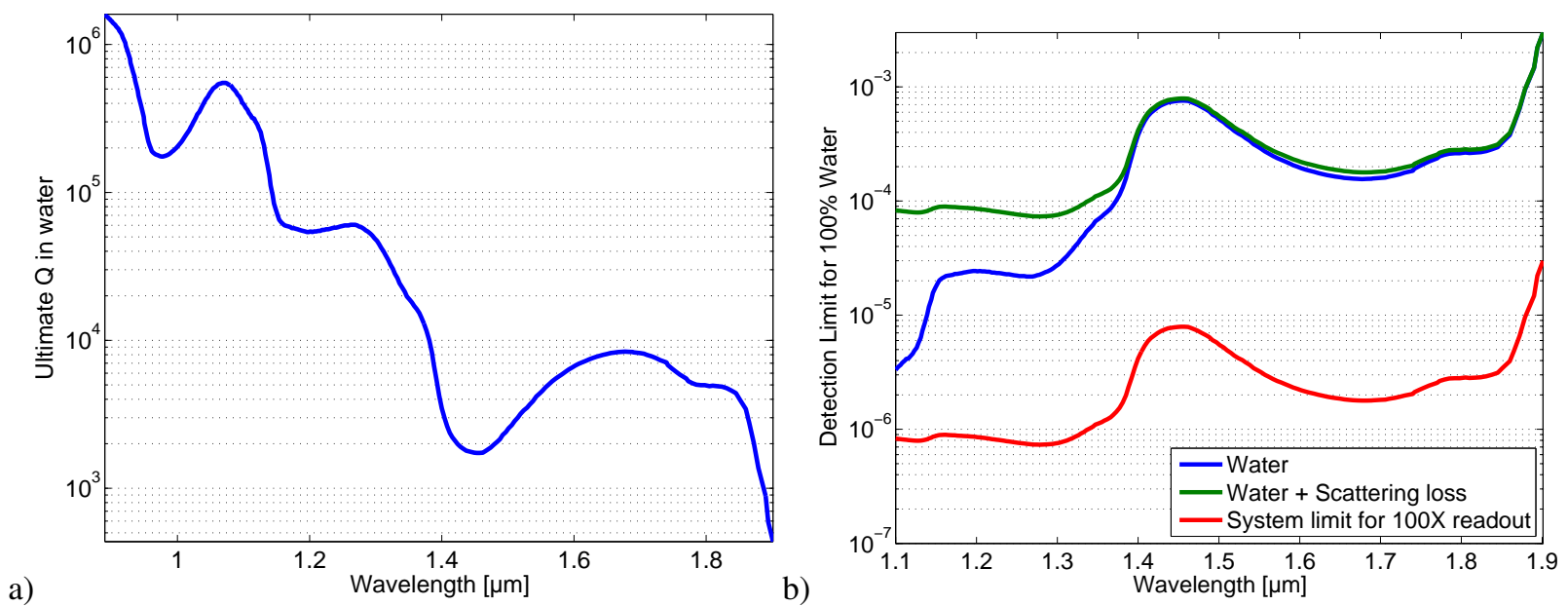

Figure 8. a) Maximum resonator Q limited by optical absorption from water. b) Highest predicted limits of detection for water absorptionlimited sensing. Waveguide scattering is subsequently added and assumed to contribute $5 \mathrm{~dB} / \mathrm{cm} \operatorname{loss}$ at $1550 \mathrm{~nm}$, and scale as $1 / \lambda^{4}$ for other wavelengths. Finally, the system detection limit is shown, assuming a wavelength readout precision $100 \mathrm{X}$ better than the resonator linewidth. Note that silicon optical absorption, not included in here, dominates over water absorption for wavelengths $<1.1 \mu \mathrm{m}$ and is negligible for wavelengths $>1.1 \mu \mathrm{m}$.

Table 1. Summary of silicon photonic sensors and their performance

Experimental results

\begin{tabular}{|c|c|c|c|c|c|}
\hline Sensor type & Geometry & Q (in water) & S [nm/RIU] & S' [/RIU] $^{\prime}$ & $\Delta n_{\min }$ \\
\hline Ring $^{41}$ & TM, 260x450nm strip & 39,299 & 135 & 0.088 & $2.9 \times 10^{-4}$ \\
Ring $^{5}$ & TE, 220x500 nm & 20,000 & 70 & 0.045 & $1.1 \times 10^{-3}$ \\
Disk & TE, 220x5 $\mu m$ & 90,000 & 27 & 0.0175 & $6.4 \times 10^{-4}$ \\
\hline
\end{tabular}

Modelling results: $1550 \mathrm{~nm}$, in water. Coupling losses not included (intrinsic resonator only).

\begin{tabular}{|c|c|c|c|c|c|c|c|c|}
\hline Sensor type & Geometry & Q & dB/cm & FSR [nm] & $\partial n_{\text {eff }} / \partial n_{\text {fluid }}$ & S & S & $\Delta n_{\min }$ \\
\hline Ring & TE, 220x500 nm strip & 106,719 & 6.9 & up to 6 & 0.152 & 56 & 0.036 & $2.57 \times 10^{-4}$ \\
Ring & TM, 220x500 nm strip & 34,500 & 22.7 & up to 6 & 0.52 & 179 & 0.116 & $2.51 \times 10^{-4}$ \\
Disk & TE, 220x2.5 $\mu m$ & $\mathbf{2 3 2 , 8 5 4}$ & $\mathbf{2 . 8}$ & $\mathbf{4 5}$ & 0.064 & 27 & 0.017 & $2.49 \times 10^{-4}$ \\
Slot & TE, 220x550/110 nm & 12,822 & 34 & up to 6 & $\mathbf{0 . 8 0}$ & $\mathbf{4 6 0}$ & 0.30 & $2.63 \times 10^{-4}$ \\
\hline
\end{tabular}

\subsection{Comparison of Modelling versus Experiments}

A summary of selected published resonator sensor results, together with the experimental and modelling results presented in this paper, are given in Table 1. Modelling of the strip waveguide ring resonator (TE and TM modes), disk resonator (TE and TM mode), and slot waveguide ring resonator (TE mode) were conducted using the methodology described in Section 2.6. Specifically, we consider an intrinsic resonator, without any out-coupling losses and with side-wall roughness scattering. The parameters of interest are the propagation loss $(\mathrm{dB} / \mathrm{cm})$ from the water absorption and radiation losses which determines the $\mathrm{Q}$ of the resonator, as well as the sensitivity $\left(\mathrm{S}, \mathrm{S}^{\prime}\right)$ which is found by determining the waveguide susceptibility $\left(\partial n_{\text {eff }} / \partial n_{\text {fluid }}\right)$. These values are used to find the intrinsic limit of detection, $\Delta n_{\min }$. Additionally, the resonator free-spectral range (FSR) is identified.

The most important observation from the modelling results is that all resonators should theoretically have the same limit of detection, under the condition of a fixed wavelength and no scattering losses. The simulated results ( $\left.\mathrm{S}^{\prime}, \mathrm{Q}, \Delta n_{\min }\right)$ for the various structures are shown in Fig. 9. As shown, all sensors have the same sensitivity of $\Delta n_{\min } \sim 2.5 \times 10^{-4}$. This, according to Eq. 5, implies that the fundamental limit of the sensor is that the sensitivity is inversely proportional to the $\mathrm{Q}$ factor. Any increase in the sensitivity (e.g., by employing slot waveguides) will simply reduce the $\mathrm{Q}$ factor by the same amount. Similarly, high-Q structures (e.g., disks) that do not interact much with water will have a lower sensitivity. 


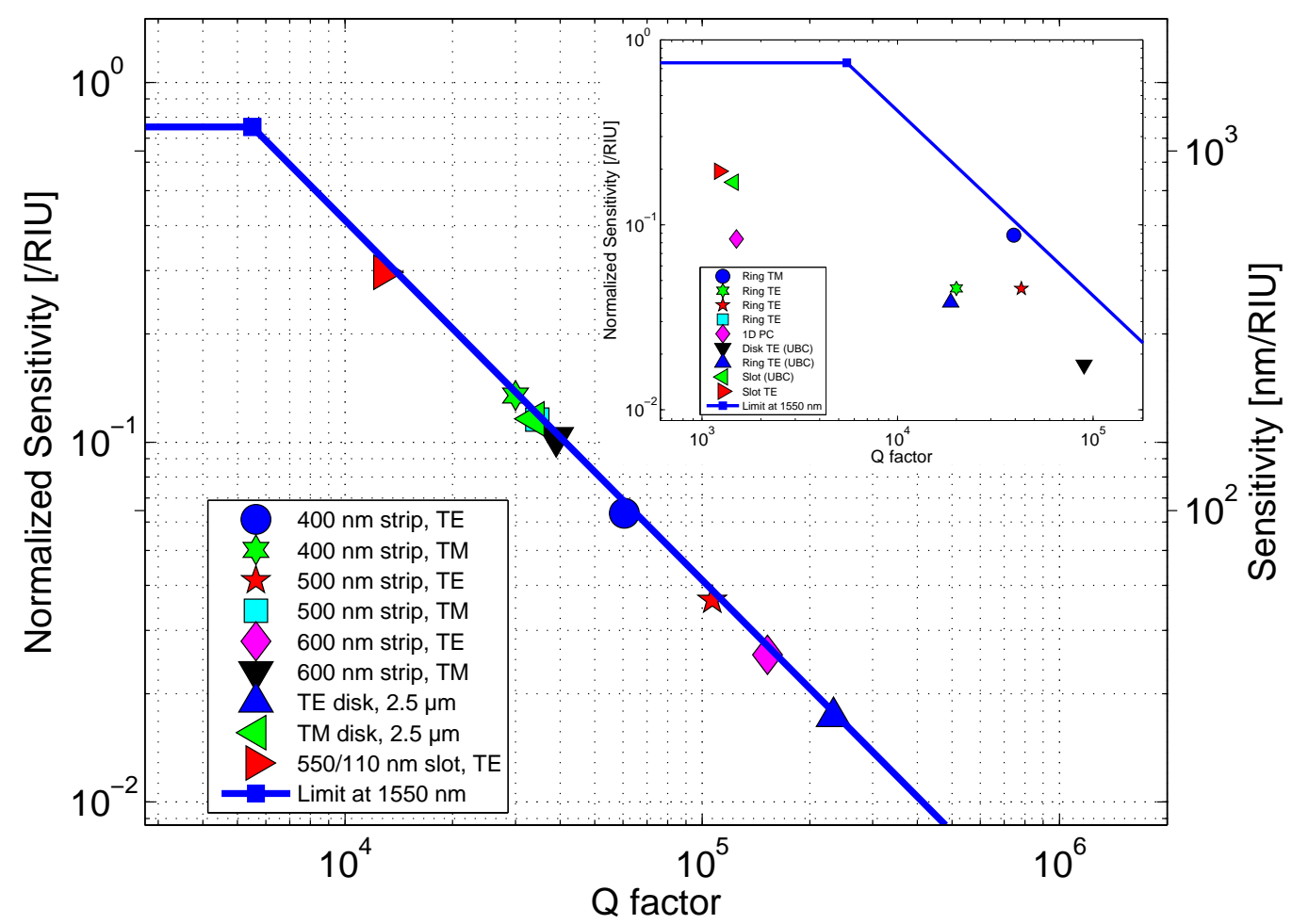

Figure 9. Summary of model results for Limits of Detection (LD) for $1550 \mathrm{~nm}$ silicon photonic biosensors, for the various configurations studied in this work (data points). The theoretical maximum based on the water absorption limit is the line, in which the single data point represents light travelling exclusively in water. From this point, two limits are drawn: a) the line to the left of the point is the maximum sensitivity where light has $100 \%$ interaction with water, namely $\partial n_{\text {eff }} / \partial n_{\text {fluid }}=1$, and b) the line diagonally down-right shows that the Q-factor limit due to the water absorption increases as the light interacts less with the water, i.e., $\partial n_{\text {eff }} / \partial n_{\text {fluid }}<1$. These limits only depend on, respectively: a) the index of refraction of the analyte, b) the optical loss of the analyte, i.e., $n(\lambda)+i k(\lambda)$. Inset: Experimental data points from Fig. 1 with the addition of the theoretical maximum lines.

To understand why different resonators have various levels of sensitivity in Fig. 1, we need to consider the additional loss mechanisms in the resonators. The dominant loss in the TE-like mode devices is the side-wall roughness scattering. As a result, devices with a strong field overlap with the sidewall will have significantly increased propagation loss, hence reduced $\mathrm{Q}$, and a reduced limit of detection. This can be quantified by comparing the total propagation losses versus losses induced by the water absorption. Ideally water absorption would dominate to achieve the highest limit of detection. In the case of the slot waveguide resonators, our measured $\mathrm{Q}$ values have been 1,450, while the intrinsic $\mathrm{Q}$ from water absorption would suggest a Q of over 12,000. Additionally, it is believed that the analyte does not penetrate the slot completely. Hence, $S^{\prime}$ is slightly reduced, Q is significantly reduced, which leads to a limit of detection, $2.4 \times 10^{-3}$, that is $10 \mathrm{X}$ lower than theoretically possible.

Meanwhile, the TE disk resonators have a limit of detection of $5.5 \times 10^{-4}$, which is only $2 \mathrm{X}$ lower than theoretically possible. This is better than the TE mode strip waveguide resonators, and is explained as follows: a) the whispering gallery mode is only confined by one waveguide side-wall, rather than the two in the strip waveguides, hence the scattering losses are reduced, b) the mode-profile has a shape that is more similar to a $600 \mathrm{~nm}$ waveguide, and it is known that wider waveguides have lower losses ${ }^{13}$ due to reduced interaction with the side-walls. These two factors contribute to a higher $\mathrm{Q}$ factor. The disadvantage is that the electric field interacts less with the analyte, hence lower sensitivity. However, a potential advantage is that the field-analyte interaction is now primarily above the disk, as opposed to on the side of the waveguides as in TE strip waveguides, which may be beneficial for biological applications where the analyte (e.g., virus) can more easily diffuse and adsorb to the top surface.

An interesting observation from Table 1 and Fig. 1 is that some of the demonstrated $1550 \mathrm{~nm}$ sensors already measure at the fundamental water absorption limit. Specifically, this was achieved in the system using TM polarization where a 
Table 2. Loss mechanisms in silicon photonic sensors. Resonators are assumed to be in isolation. If they were critically coupled, the total $\mathrm{Q}$ would be reduced by $2 \mathrm{X}$, hence the limit of detection would be reduced by $2 \mathrm{X}$.

\begin{tabular}{|c|c|c|l|l|l|l|l|}
\hline Sensor & $Q_{\text {scattering }}$ & $Q_{\text {water }}$ & $\begin{array}{l}Q_{\text {scattering }} \\
Q_{\text {water }}\end{array}$ & $\begin{array}{l}\mathrm{dB} / \mathrm{cm} \\
(\text { scattering) }\end{array}$ & $\begin{array}{l}\mathrm{dB} / \mathrm{cm} \\
\text { (water) }\end{array}$ & $\begin{array}{l}\mathrm{dB} / \mathrm{cm} \\
\text { (total) }\end{array}$ & $\begin{array}{l}\text { Predicted } \\
\Delta n_{\min }\end{array}$ \\
\hline Ideal, water only & $\infty$ & 5500 & $\infty$ & 0 & 43 & 43 & $2.4 \times 10^{-4}$ \\
\hline Ring TE, 220x500 nm strip & 258,000 & 106,719 & 2.42 & 3 & 6.9 & 9.9 & $3.7 \times 10^{-4}$ \\
Ring TE, 220x500 nm strip & 147,000 & 106,719 & 1.4 & $5^{58}$ & 6.9 & 11.9 & $4.5 \times 10^{-4}$ \\
Ring TE, 220x500 nm strip & 43,000 & 106,719 & 0.40 & 18 & 6.9 & 24.9 & $9.1 \times 10^{-4}$ \\
Ring TM, 220x500 nm strip & 147,000 & 34,500 & 4.3 & 5 & 22.7 & 27.7 & $3.1 \times 10^{-4}$ \\
Disk TE, 220x2.5 $\mu m$ & 124,000 & 232,854 & 0.53 & - & - & - & $7.3 \times 10^{-4}$ \\
Slot TE, 220x550/110 nm & 58,643 & 12,822 & 4.6 & $12^{7}$ & 34 & 46 & $3.2 \times 10^{-4}$ \\
Slot TE, 220x550/110 nm & 1,450 & 12,822 & 0.12 & $500^{7}$ & 34 & 534 & $2.6 \times 10^{-3}$ \\
\hline
\end{tabular}

large portion of the light is above the waveguide. ${ }^{41}$ In this case, the water absorption dominates, and the limit of detection almost matches that predicted in Fig. 9.

In summary, the best device (as quantified by the limit of detection) would have no loss (except for water, and some for sensing itself via couplers) and the light would resonate primarily in the sensing fluid. In the case where the waveguide scattering and radiation losses can be reduced to below the water absorption losses, operating at different wavelengths can also be considered as shown in Fig. 8. With present fabrication technology (scattering loss limited), only the TM mode resonators would benefit from a reduced water absorption from a change in wavelength.

It is interesting to consider whether there exist methods of beating this limit. For example, high group index structures such as Bragg gratings, photonic crystals, or plasmonic devices, will enable the light to travel more slowly through the water hence potentially have sensitivities higher than predicted by Eq. 3. However, this comes together with correspondingly higher optical loss, hence reduced Q. Indeed, SPR prism-coupled sensors have much higher sensitivity, albeit with a very broad linewidth (e.g., over $100 \mathrm{~nm}$ ), and ultimately their limits of detection are comparable to these considered here (the different wavelength used in SPR prevents the direct comparison with this analysis).

\subsection{Readout}

Until now, the discussion has focused on a limit of detection determined by the linewidth of the resonator. This was done specifically to compare the intrinsic performance of difference resonators, independent of the signal processing and detection methods. Measurement of the resonant wavelength typically involves measuring a spectrum. How the resonant wavelength is measured will impact the precision. This is particularly true when the spectrum contains amplitude (and wavelength) noise. Curve fitting the spectrum to an analytic transfer function is a method in which the algorithm takes into account contributions from the entire spectrum, rather than simply finding the peak. Similar to averaging, this improves the eventual signal-to-noise ratio by $\sqrt{N}$, where $N$ is the number of data points in the spectrum. If the noise is low enough, this technique can result in an interpolation of the resonant wavelength and achieve a wavelength resolution even below the original data sample spacing. Optical spectrum processing has been applied to silicon photonic sensors, and has resulted in a wavelength measurement precision that is $10 \mathrm{~s}$ to $100 \mathrm{~s}$ of times smaller than the resonator linewidth. ${ }^{37}$ For example, the Genolyte system achieves a $3 \sigma$ wavelength measurement noise of $0.2 \mathrm{pm} .{ }^{10}$ Compare this to a typical 500x220 nm strip-waveguide ring resonator with a $\mathrm{Q}$ of 30,000 in water at $1550 \mathrm{~nm}$, with a linewidth of $\Delta \lambda_{1 / 2}=50 \mathrm{pm}$, this implies an enhancement factor of over $250 \mathrm{X}$ in the spectrum readout as compared to the resonator linewidth used in the intrinsic limit of detection defined in Eq. 5.

The limit of detection analysis can also be applied to temperature sensors. Assuming spectral sweep measurement can result in a 100X higher wavelength measurement precision than the resonator linewidth (typical ${ }^{37}$ ), and for a resonator with a $\mathrm{Q}=100,000$, and a sensitivity of $S=0.08 \mathrm{~nm} / K{ }^{49}$ the thermal detection limit is comparable to thermistors:

$$
D L=\frac{\lambda}{100 \cdot Q \cdot S}=\frac{1550 \times 10^{-9}}{100 \cdot 10^{6} \cdot 0.08 \times 10^{-9}}=2 m K
$$




\subsection{Biology Applications}

We have used the ring resonator sensors for biosensing using Human Factor IX and its corresponding antibody ${ }^{59}$ and verify that the devices are able to sense both the protein attachment during functionalization and the protein-antibody binding reaction. The silicon photonic sample preparation begins by cleaning it in a Piranha solution at $80^{\circ} \mathrm{C}$ for $20 \mathrm{~min}$, then removing the Si native oxide on top of the waveguides using a hydrofluoric acid etchant for $20 \mathrm{~s}$. The samples were then thoroughly rinsed in DI water, activated in an air plasma, and incubated in a 1\% APTMS solution (94\% Ethanol, $5 \%$ DI water, APTMS (97\%, Sigma-Aldrich)) for $3 \mathrm{~h}$. The Human Factor IX was covalently attached with a NHS/EDC chemistry to the amine group of the APTMS linker molecule (0.1 M N-Hydroxysuccinimide, NHS and 0.4 M 1-Ethyl-3(3- dimethylaminopropyl)carbodiimide, EDC, in equal parts, and $500 \mu \mathrm{g} / \mathrm{mL}$ Human Factor IX). The different deposition steps were characterized by X-ray photoelectron spectroscopy (XPS) and ellipsometry. The thickness of the APTMS was approximately $8 \stackrel{\AA}{A}$ and the thickness of the protein layer was approximately $4 \mathrm{~nm}$. The PDMS microfludic channels were then aligned and reversibly bonded to the SOI substrate as described in Section 2.1.

The resonance peak shift is then monitored as a function of time, Fig. 10 while functionalizing the racetrack with the protein Human Factor IX in a Phosphate buffered saline (PBS) solution. The position of the resonance peak corresponding to the racetrack in the control channel (which remained filled with PBS) is also monitored as a function of time in order to quantify measurement noise and thermal drift ( $3 \sigma$ variation of $2.8 \mathrm{pm})$. The initial larger shift is caused by a combined effect of a bulk refractive index change of the protein solution and the deposition of a protein layer on top of the waveguide. After switching back to PBS, $\Delta \lambda_{P}$ is then a measurement of the mass change on the waveguides surface.

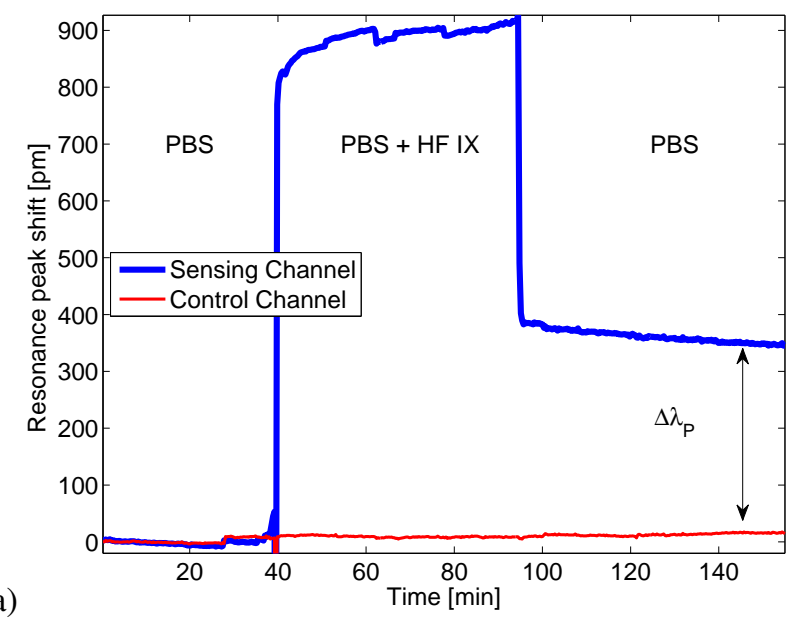

b)

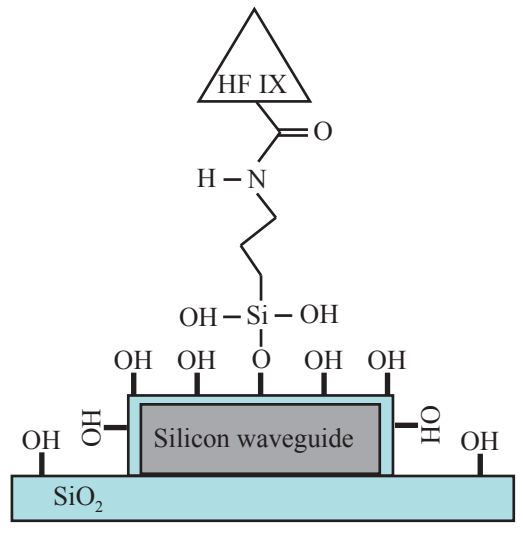

Figure 10. a) Ring resonator resonance peak shift during attachment of the protein Human Factor IX $(56 \mathrm{kDa}, 500 \mu g / m L)$. The values for both the sensing (blue) and control (red) channels are plotted. The difference in peak location $\left(\Delta \lambda_{P}\right)$ before and after (both in PBS) is a measurement of the mass change due to the adsorption of the HF IX protein on the sensor. b) Diagram of the attached molecules on the silicon waveguide.

\section{CONCLUSION}

Sensors operating in aqueous media are fundamentally limited by the optical absorption of water. In the cases where the water (or other sensing media) absorption is dominant, all sensor geometries (ring resonators based on strip or slot waveguides, disks, Bragg grating cavities, 1D photonic crystals, etc) and light polarizations (TE-like, TM-like) will theoretically offer the same performance in terms of limit of detection. The differences between sensor configurations is important where other loss mechanisms are present, such as waveguide scattering loss, waveguide material absorption, or resonator radiation loss. In these cases, it is important to minimize the ratio of other losses relative to analyte optical absorption losses. In particular, increasing the analyte absorption by resorting to TM modes, and moving the optical field away from the rough waveguide sidewall, improves this ratio and allows the sensor to approach the fundamental limits of detection. The structures that are most amenable to reaching the theoretical limit are in decreasing order: TM waveguides, disks, and TE waveguides. Slot waveguides and 1D photonic crystals are the furthest away from reaching fundamental limits. 
Of the sensors surveyed in this work, we find that many silicon photonics sensors are near the fundamental limit of detection. Hence, future sensor development should focus on additional system functionality and ease-of-use, e.g., very large array operation, low-cost point-of-care devices, electronic readout, further improvement in readout sensitivity and noise considerations, etc. Additionally, silicon photonic resonator sensor technology has matured to the point where commercially available systems are available ${ }^{10}$ and enable complex biological experimentation research to take place. ${ }^{29}$ The next steps will be for this technology to move to clinical, hospital, and point-of-care applications.

\section{ACKNOWLEDGMENTS}

The author would like to thank CMC Microsystems for enabling the fabrication of the chips via IMEC-ePIXfab and for providing the Design Workshop Technologies and Mentor Graphics Pyxis layout software packages, and to Lumerical Solutions, Inc. for supporting the CMC-UBC Silicon Nanophotonics Fabrication course and workshop by providing FDTD and MODE Solutions software. Funding for this research was provided by NSERC. SAS and DMR gratefully acknowledge support from US NSF CBET (award no. 0930411). Finally, the authors are grateful to Michael Hochberg, Mike Gould, and Jim Kirk at the University of Washington, and Miguel A.G. Torres at UBC for many useful discussions. We thank Ralph Azucena for the laser automation.

\section{REFERENCES}

1. Washburn, A. L., Gunn, L. C., and Bailey, R. C., "Label-free quantitation of a cancer biomarker in complex media using silicon photonic microring resonators," Analytical Chemistry 81(22), 9499-9506 (2009). doi: 10.1021/ac902006p.

2. White, I. and Fan, X., "On the performance quantification of resonant refractive index sensors," Optics Express 16(2), 1020 (2008).

3. Yang, G. M., White, I. M., and Fan, X. D., "An opto-fluidic ring resonator biosensor for the detection of organophosphorus pesticides," Sensors and Actuators B: Chemical 133(1), 105-112 (2008).

4. Passaro, V. M. N., Dell'Olio, F., Ciminelli, C., and Armenise, M. N., "Efficient chemical sensing by coupled slot soi waveguides," Sensors 9(2), 1012-1032 (2009).

5. De Vos, K., Bartolozzi, I., Schacht, E., Bienstman, P., and Baets, R., "Silicon-on-insulator microring resonator for sensitive and label-free biosensing," Optics Express 15(12), 7610-7615 (2007).

6. Ramachandran, A., Wang, S., Clarke, J., Ja, S. J., Goad, D., Wald, L., Flood, E. M., Knobbe, E., Hryniewicz, J. V., Chu, S. T., Gill, D., Chen, W., King, O., and Little, B. E., "A universal biosensing platform based on optical micro-ring resonators," Biosensors and Bioelectronics 23(7), 939-944 (2008).

7. Claes, T., Molera, J. G., De Vos, K., Schacht, E., Baets, R., and Bienstman, P., "Label-free biosensing with a slotwaveguide-based ring resonator in silicon on insulator," IEEE Photonics Journal 1(3), 197-204 (2009).

8. Carlborg, C. F., Gylfason, K. B., Kazmierczak, A., Dortu, F., Polo, M. J. B., Catala, A. M., Kresbach, G. M., Sohlstrom, H., Moh, T., Vivien, L., Popplewell, J., Ronan, G., Barrios, C. A., Stemme, G., and van der Wijngaart, W., "A packaged optical slot-waveguide ring resonator sensor array for multiplex label-free assays in labs-on-chips," Lab on a chip 10(3), 281-290 (2010).

9. Luchansky, M. and Bailey, R., "Silicon photonic microring resonators for quantitative cytokine detection and t-cell secretion analysis," Analytical chemistry 82(5), 1975-1981 (2010).

10. Iqbal, M., Gleeson, M., Spaugh, B., Tybor, F., Gunn, W., Hochberg, M., Baehr-Jones, T., Bailey, R., and Gunn, L., "Label-free biosensor arrays based on silicon ring resonators and high-speed optical scanning instrumentation," IEEE Journal of Selected Topics in Quantum Electronics 16(3), 654-661 (2010).

11. Fan, X. D., White, I. M., Shopoua, S. I., Zhu, H. Y., Suter, J. D., and Sun, Y. Z., "Sensitive optical biosensors for unlabeled targets: A review," Analytica Chimica Acta 620(1-2), 8-26 (2008).

12. Passaro, V. M. N., Dell'Olio, F., Casamassima, B., and De Leonardis, F., "Guided-wave optical biosensors," Sensors 7(4), 508-536 (2007).

13. Vlasov, Y. and McNab, S., "Losses in single-mode silicon-on-insulator strip waveguides and bends," Optics Express 12(8), 1622-1631 (2004).

14. Densmore, A., Xu, D., Waldron, P., Janz, S., Cheben, P., Lapointe, J., Delge, A., Lamontagne, B., Schmid, J., and Post, E., "A silicon-on-insulator photonic wire based evanescent field sensor," IEEE Photonics Technology Letters 18(23), 2520-2522 (2006). 
15. Baehr-Jones, T., Hochberg, M., Walker, C., and Scherer, A., "High-Q optical resonators in silicon-on-insulator-based slot waveguides," Applied Physics Letters 86(8), - (2005).

16. Barrios, C. A., Gylfason, K. B., Sanchez, B., Griol, A., Sohlstrom, H., Holgado, M., and Casquel, R., "Slot-waveguide biochemical sensor," Optics Letters 32(21), 3080-3082 (2007).

17. Dell'Olio, F. and Passaro, V. M. N., "Optical sensing by optimized silicon slot waveguides," Optics Express 15(8), 4977-4993 (2007).

18. Barrios, C. A., Banuls, M. J., Gonzalez-Pedro, V., Gylfason, K. B., Sanchez, B., Griol, A., Maquieira, A., Sohlstrom, H., Holgado, M., and Casquel, R., "Label-free optical biosensing with slot-waveguides," Optics Letters 33(7), 708710 (2008).

19. Keivani, H. and Kargar, A., "Bending efficiency of bent multiple-slot waveguides," Chinese Physics Letters 26(12), - (2009).

20. Boyd, R. and Heebner, J., "Sensitive disk resonator photonic biosensor," Applied Optics 40(31), $5742-5747$ (2001).

21. Armani, A., Kulkarni, R., Fraser, S., Flagan, R., and Vahala, K., "Label-free, single-molecule detection with optical microcavities," Science 317(5839), 783 (2007).

22. Jeong, H., Lee, S., Sung, G., and Shin, J., "Design and fabrication of $\mathrm{Tb}^{3+}$-doped silicon oxy-nitride microdisk for biosensor applications," IEEE Photonics Technology Letters 23(2), 88-90 (2011).

23. Mandal, S. and Erickson, D., "Nanoscale optofluidic sensor arrays," Optics Express 16(3), 1623 (2008).

24. Prabhathan, P., Murukeshan, V., Jing, Z., and Ramana, P., "Compact SOI nanowire refractive index sensor using phase shifted bragg grating," Optics Express 17(17), 15330-15341 (2009).

25. Arce, C., De Vos, K., Claes, T., Komorowska, K., Van Thourhout, D., and Bienstman, P., "Silicon-on-insulator microring resonator sensor integrated on an optical fiber facet," IEEE Photonics Technology Letters 23(13) (2011).

26. Monat, C., Domachuk, P., and Eggleton, B. J., "Integrated optofluidics: A new river of light," Nature Photonics 1(2), 106-114 (2007).

27. Gylfason, K. B., Carlborg, C. F., Kazmierczak, A., Dortu, F., Sohlstrom, H., Vivien, L., Barrios, C. A., van der Wijngaart, W., and Stemme, G., "On-chip temperature compensation in an integrated slot-waveguide ring resonator refractive index sensor array," Optics Express 18(4), 3226-3237 (2010).

28. Xu, D. X., Densmore, A., Delage, A., Waldron, P., McKinnon, R., Janz, S., Lapointe, J., Lopinski, G., Mischki, T., Post, E., Cheben, P., and Schmid, J. H., "Folded cavity soi microring sensors for high sensitivity and real time measurement of biomolecular binding," Optics Express 16(19), 15137-15148 (2008).

29. Kirk, J., Fridley, G., Chamberlain, J., Christensen, E., Hochberg, M., and Ratner, D., "Multiplexed inkjet functionalization of silicon photonic biosensors," Lab on a Chip (2011).

30. Narayanaswamy, R. and Wolfbeis, O., [Optical sensors: industrial, environmental and diagnostic applications], vol. 1, Springer Verlag (2004).

31. Bailey, R. C., Washburn, A. L., Qavi, A. J., Iqbal, M., Gleeson, M., Tybor, F., and Gunn, L. C., "A robust silicon photonic platform for multiparameter biological analysis," 72200N-72200N-6 (2009).

32. Adam, B., Qu, Y., Davis, J., Ward, M., Clements, M., Cazares, L., Semmes, O., Schellhammer, P., Yasui, Y., and Feng, Z., "Serum protein fingerprinting coupled with a pattern-matching algorithm distinguishes prostate cancer from benign prostate hyperplasia and healthy men," Cancer Research 62(13), 3609 (2002).

33. Washburn, A., Luchansky, M., Bowman, A., and Bailey, R., "Quantitative, label-free detection of five protein biomarkers using multiplexed arrays of silicon photonic microring resonators," Anal Chem 82(1), 69-72 (2009).

34. Qavi, A. and Bailey, R., "Multiplexed detection and labelfree quantitation of micrornas using arrays of silicon photonic microring resonators," Angewandte Chemie International Edition 49(27), 4608-4611 (2010).

35. Yan, R., Kingry, L. C., Slayden, R. A., and Lear, K. L., "Immunoassay demonstration using a local evanescent array coupled biosensor," 75590D-75590D-7 (2010).

36. Hochberg, M. and Baehr-Jones, T., "Towards fabless silicon photonics," Nature Photonics 4(8), 492-494 (2010).

37. Yoshie, T., Tang, L., and Su, S., "Optical microcavity: Sensing down to single molecules and atoms," Sensors 11(2), 1972-1991 (2011).

38. Ackert, J., Doylend, J., Logan, D., Jessop, P., Vafaei, R., Chrostowski, L., and Knights, A., "Defect-mediated resonance shift of silicon-on-insulator racetrack resonators," Optics Express 19(13), 11969-11976 (2011). 
39. Baehr-Jones, T., Hochberg, M., Walker, C., Chan, E., Koshinz, D., Krug, W., and Scherer, A., "Analysis of the tuning sensitivity of silicon-on-insulator optical ring resonators," IEEE Journal of Lightwave Technology 23(12), 4215 (2005).

40. Markov, D., Begari, D., and Bornhop, D., "Breaking the 10-7 barrier for ri measurements in nanoliter volumes," Analytical chemistry 74(20), 5438-5441 (2002).

41. Xu, D., Vachon, M., Densmore, A., Ma, R., Janz, S., Delge, A., Lapointe, J., Cheben, P., Schmid, J., Post, E., et al., "Real-time cancellation of temperature induced resonance shifts in soi wire waveguide ring resonator label-free biosensor arrays," Optics Express 18(22), 22867-22879 (2010).

42. Lukosz, W., "Principles and sensitivities of integrated optical and surface plasmon sensors for direct affinity sensing and immunosensing," Biosensors and Bioelectronics 6(3), 215-225 (1991).

43. "http://www.biacore.com/,"

44. Unger, M., Chou, H., Thorsen, T., Scherer, A., and Quake, S., "Monolithic microfabricated valves and pumps by multilayer soft lithography," Science 288(5463), 113 (2000).

45. Ksendzov, A. and Lin, Y., "Integrated optics ring-resonator sensors for protein detection," Optics Letters 30(24), 3344-3346 (2005).

46. Xia, Y. N. and Whitesides, G. M., "Soft lithography," Annual Review of Materials Science 28, 153-184 (1998). 110TC Times Cited:1318 Cited References Count:184.

47. DowChemical, "Optim glycerine refractive index," (2011).

48. Flueckiger, J., Grist, S., Bisra, G., Chrostowski, L., and Cheung, K., "Cascaded silicon-on-insulator microring resonators for the detection of biomolecules in PDMS microfluidic channels," Proceedings of SPIE 7929, 79290I (2011).

49. Rouger, N., Chrostowski, L., and Vafaei, R., "Temperature effects on silicon-on-insulator (SOI) racetrack resonators: a coupled analytic and 2D finite difference approach," IEEE Journal of Lightwave Technology 28(9), 1380-1391 (2010).

50. "http://www.lumerical.com/mode/," Lumerical Solutions MODE.

51. Wang, X., Shi, W., Grist, S., Yun, H., and Chrostowski, N. A. F. J., "Narrow-band transmission filter using phaseshifted bragg gratings in SOI waveguide," IEEE Photonics Conference, ThZ1 (10/2011 2011).

52. Borselli, M., Johnson, T., and Painter, O., "Beyond the rayleigh scattering limit in high-q silicon microdisks: theory and experiment," Opt. Express 13, 1515-1530 (Mar 2005).

53. Morand, A., Zhang, Y., Martin, B., Huy, K. P., Amans, D., Benech, P., Verbert, J., Hadji, E., and Fédéli, J.-M., "Ultra-compact microdisk resonator filters on soi substrate," Opt. Express 14, 12814-12821 (Dec 2006).

54. Soltani, M., Li, Q., Yegnanarayanan, S., and Adibi, A., "Toward ultimate miniaturization of high Q silicon travelingwave microresonators," Optics Express 18(19), 19541-19557 (2010).

55. "http://www.lumerical.com/fdtd/," Lumerical Solutions FDTD .

56. Homola, J., Koudela, I., and Yee, S., "Surface plasmon resonance sensors based on diffraction gratings and prism couplers: sensitivity comparison," Sensors and Actuators B: Chemical 54(1-2), 16-24 (1999).

57. Kou, L., Labrie, D., and Chylek, P., "Refractive indices of water and ice in the $0.65-$ to $2.5-\mu \mathrm{m}$ spectral range," Applied Optics 32, 3531-3540 (Jul 1993).

58. Shi, W., Vafaei, R., Torres, M., Jaeger, N., and Chrostowski, L., "Design and characterization of microring reflectors with a waveguide crossing," Optics Letters 35(17), 2901-2903 (2010).

59. Flueckiger, J., Grist, S. M., Ouellet, E., Chrostowski, L., and Cheung, K. C., "Label-free biosensing using cascaded silicon-on-insulator micro-racetrack resonators integrated with PDMS microfluidic channels," International Conference on Miniaturized Systems for Chemistry and Life Sciences ( $\mu$ TAS), 565-567 (Oct. 2011). 